Annals of Pure and Applied Mathematics

Vol. 14, No. 2, 2017, 321-326

ISSN: 2279-087X (P), 2279-0888(online)

Published on 30 September 2017

www.researchmathsci.org

DOI: http://dx.doi.org/10.22457/apam.v14n2a15

\begin{tabular}{l}
\hline$\overline{\text { Pnals of }}$ Pupre and Applied \\
Mathematics
\end{tabular}

\title{
On a New Fractional Integral Transform and its Applications
}

\author{
Pravinkumar V. Dole $^{l}$ and S.K.Panchal ${ }^{2}$ \\ Department of Mathematics \\ Dr. Babasaheb Ambedkar Marathwada University \\ Aurangabad-431004 (M.S.) India \\ Email: ${ }^{1}$ pvasudeo.dole@gmail.com, ${ }^{2}$ drpanchalsk@gmail.com \\ ${ }^{1}$ Corresponding author
}

Received 23 August 2017; accepted 12 September 2017

\begin{abstract}
In this paper, we have introduced the new fractional integral transform and proved some of its basic properties. Then obtained new fractional integral transform of some elementary functions and conformable derivative. Further, we define new convolution operation, proved convolution theorem for new fractional integral transform and commutative semi group property of new convolution operation. At last the new fractional integral transform is applied to obtain the solutions of diffusion equation and heat-transfer problem involving conformable derivative.
\end{abstract}

Keywords: New fractional integral transforms; conformable derivative; convolution.

AMS Mathematics Subject Classification (2010): 26A33, 42A38, 34A08

\section{Introduction}

The concept of integral transforms is originated from the Fourier integral formula. The importance of integral transforms is that they provide powerful operational method for solving initial and boundary value problems. The operational calculus of integral transform is used to solve the differential, fractional differential and integral equations arising in applied mathematics, mathematical physics and Engineering science etc. [2, 3, 7, 8, 9, 10, 11]. In recent years, fractional order differential equations have become an important tool in mathematical modeling [1]. The idea of fractional is natural. $\partial u / \partial x$ and $\left(\partial^{2} u\right) /\left(\partial x^{2}\right)$ exist, $\left(\partial^{1.5} u\right) /\left(\partial x^{1.5}\right)$ maybe exists too. Fractional equations can be used to describe some physical phenomena more accurately than the classical integer order differential equation [6]. Fractional differential equations (FDEs) provide a powerful instrument for the description of memory and hereditary properties of different substances. The fractional diffusion equations play an important role in dynamical systems of semi-conductor research, hydrogeology, bioinformatics, finance [5]. Khalil et al. defined conformable fractional derivative in [4] as given below.

Let $f \in C^{n}(0, \infty), \alpha \in(p-1, p]$ for all $p \in \mathbb{N}$ and $\lceil\alpha\rceil$ denote the smallest integer greater than or equal to $\alpha$. The conformable fractional derivative of order $\alpha$ denoted by $T_{\alpha}[f(t)]$ is defined as, 
Pravinkumar V. Dole and S.K.Panchal

$$
\begin{aligned}
T_{\alpha}[f(t)] & =\lim _{\varepsilon \rightarrow 0} \frac{f^{(\lceil\alpha\urcorner-1)}\left(t+\varepsilon t^{(「 \alpha\urcorner-\alpha)}\right)-f^{(\ulcorner\alpha\urcorner-1)}(t)}{\varepsilon} . \\
& =t^{(\lceil\alpha\urcorner-\alpha)} f^{(\lceil\alpha\urcorner)}(t)
\end{aligned}
$$

In the next section we define new fractional integral transform. Also we derive some of its basic properties, obtain new fractional integral transform of conformable derivative and some functions. Define convolution operation, proved convolution theorem and semigroup property.

\section{New fractional integral transform}

Definition 2.1. Let Set 'A' define as,

$$
\begin{gathered}
A=\left\{f \in \mathcal{L}^{p}\left(\mathbb{R}^{+}\right) /\|f(t)\|_{p, \alpha}=\int_{0}^{\infty}\left|t^{\alpha-p} f(t)\right| d t \leq M ;\right. \\
\text { for some } M>0, \forall p \in \mathbb{N} \text { and } \alpha \in(p-1, p]\} .
\end{gathered}
$$

The new fractional integral transform of a function $f \in A$ of order $\alpha \in(0,1]$ is define as,

$$
\mathcal{P}_{\alpha}[f(t)](s)=F_{\alpha}(s)=\int_{0}^{\infty} e^{\frac{-i s t^{\alpha}}{\alpha}} t^{\alpha-1} f(t) d t
$$

where $\mathcal{P}_{\alpha}$ is new fractional integral transform operator and $s \in \mathbb{R}$.

Theorem 2.1. Let $f \in A$ then new fractional integral transform $\mathcal{P}_{\alpha}[f(t)](s)=F_{\alpha}(s)$ is bounded and continuous for all $s \in \mathbb{R}$.

Proof: It follows from the definition of new fractional integral transform that we have

$$
\left|F_{\alpha}(s)\right| \leq \int_{0}^{\infty}\left|e^{\frac{-i s t^{\alpha}}{\alpha}}\right| t^{\alpha-1} f(t)\left|d t \leq \int_{0}^{\infty}\right| t^{\alpha-1} f(t) \mid d t<M, \text { for } M>0
$$

$$
\begin{aligned}
& \left|F_{\alpha}(s+h)-F_{\alpha}(s)\right|=\left|\int_{0}^{\infty}\left(e^{\frac{-i(s+h) t^{\alpha}}{\alpha}}-e^{\frac{-i s t^{\alpha}}{\alpha}}\right) t^{\alpha-1} f(t) d t\right| \\
& \leq \int_{0}^{\infty}\left|e^{\frac{-i h t^{\alpha}}{\alpha}}-1\right|\left|t^{\alpha-1} f(t)\right| d t \leq 2 \int_{0}^{\infty}\left|t^{\alpha-1} f(t)\right| d x<M .
\end{aligned}
$$

Since $\lim _{h \rightarrow 0}\left|e^{\frac{-i h t^{\alpha}}{\alpha}}-1\right| \rightarrow 0$ for all $t \in \mathbb{R}^{+}$, and hence

This shows that $F_{\alpha}(s)$ is a continuous.

$$
\lim _{h \rightarrow 0}\left|F_{\alpha}(s+h)-F_{\alpha}(s)\right|=\lim _{h \rightarrow 0} \int_{0}^{\infty}\left|e^{\frac{-i h t^{\alpha}}{\alpha}}-1\right|\left|t^{\alpha-1} f(t)\right| d t \rightarrow 0 .
$$

Theorem 2.2. Let $f, g \in A, \mathcal{P}_{\alpha}[f(t)](s)=F_{\alpha}(s), \mathcal{P}_{\alpha}[g(t)](s)=G_{\alpha}(s)$ and $a, b$ are any scalar then the following properties can be easily proved,

$$
\begin{array}{ll}
\text { (a) } & \mathcal{P}[a f(t)+b g(t)](s)=a F_{\alpha}(p)+b G_{\alpha}(s) \\
\text { (b) } & \mathcal{P}[f(a t)](s)=\frac{1}{a^{\alpha}} F_{\alpha}\left(\frac{s}{a^{\alpha}}\right) \\
\text { (c) } & \mathcal{P}\left[e^{\frac{i a t^{\alpha}}{\alpha}} f(t)\right](s)=F_{\alpha}(s-a) \\
\text { (d) } & \mathcal{P}\left[f(t) \cos \frac{a x^{\alpha}}{\alpha}\right](s)=\frac{1}{2}\left[F_{\alpha}(s-a)+F_{\alpha}(s+a)\right] \\
\text { (e) } & \mathcal{P}\left[f(t) \sin \frac{a x^{\alpha}}{\alpha}\right](s)=\frac{1}{2 i}\left[F_{\alpha}(s-a)-F_{\alpha}(s+a)\right] .
\end{array}
$$


On a New Fractional Integral Transform and its Applications

Theorem 2.3. The new fractional integral transform of conformable fractional derivative of $f \in \mathcal{L}^{1}\left(\mathbb{R}^{+}\right)$of order $\alpha \in(0,1]$ is given by

$$
\mathcal{P}_{\alpha}\left[T_{\alpha}(f(t))\right](s)=i s F_{\alpha}(s)-f(0),
$$

where $T_{\alpha}(f(t)) \in A$ and $\mathcal{P}_{\alpha}[f(t)](s)=F_{\alpha}(s)$.

Proof: Form the definition, we have

$$
\begin{aligned}
& \mathcal{P}_{\alpha}\left[T_{\alpha} f(t)\right](s)=\int_{0}^{\infty} e^{\frac{-i s t^{\alpha}}{\alpha}} t^{\alpha-1} t^{1-\alpha} f^{\prime}(t) d t \\
& =-f(0)+i s \int_{0}^{\infty} e^{\frac{-i s t^{\alpha}}{\alpha}} t^{\alpha-1} f(t) d t=(i s) F_{\alpha}(s)-f(0) .
\end{aligned}
$$

(i) The new fractional integral transform of $e^{\frac{-a t^{\alpha}}{\alpha}}$ for $a \in \mathbb{R}$ as

$$
\mathcal{P}_{\alpha}\left[e^{\frac{-a t^{\alpha}}{\alpha}}\right](s)=\int_{0}^{\infty} e^{\frac{-(i s+a) t^{\alpha}}{\alpha}} t^{\alpha-1} d t=\int_{0}^{\infty} e^{\frac{-(i s+a) x}{\alpha}} d x=\frac{1}{i s+a} .
$$

(ii) The new fractional integral transform of $\cosh \left(\frac{a t^{\alpha}}{\alpha}\right)$ for $a \in \mathbb{R}$ as

$$
\begin{aligned}
& \mathcal{P}_{\alpha}\left[\cosh \left(\frac{a t^{\alpha}}{\alpha}\right)\right](s)=\int_{0}^{\infty} e^{\frac{-i s t^{\alpha}}{\alpha}} t^{\alpha-1} \cosh \left(\frac{a t^{\alpha}}{\alpha}\right) d t \\
& =\frac{1}{2} \int_{0}^{\infty}\left(e^{\frac{-(i s-a) t^{\alpha}}{\alpha}}-e^{\frac{-(i s+a) t^{\alpha}}{\alpha}}\right) t^{\alpha-1} d t \\
& =\frac{1}{2} \int_{0}^{\infty}\left(e^{\frac{-(i s-a) x}{\alpha}}-e^{\frac{-(i s+a) x}{\alpha}}\right) d x=\frac{i s}{(i s)^{2}-a^{2}} .
\end{aligned}
$$

Now for $f, g \in A$, we define the convolution $(f * g)$ as

where

$$
\begin{aligned}
(f * g)(t) & =\int_{0}^{t} e^{\frac{-i s}{\alpha}\left(x^{\alpha}-t^{\alpha}\right)} e^{\frac{-i s}{\alpha}(t-x)^{\alpha}}(t-x)^{\alpha-1} x^{\alpha-1} t^{1-\alpha} f(x) g(t-x) d x \\
& =e^{\frac{i s}{\alpha} t^{\alpha}} t^{1-\alpha}(\hat{f} * \hat{g})(t),
\end{aligned}
$$

$$
(\hat{f} * \hat{g})(t)=\int_{0}^{t} \hat{f}(x) \hat{g}(t-x) d x,
$$

for $\hat{f}(x)=e^{\frac{-i s}{\alpha} x^{\alpha}} x^{\alpha-1} f(x)$ and $\hat{g}(t-x)=e^{\frac{-i s}{\alpha}(t-x)^{\alpha}}(t-x)^{\alpha-1} g(t-x)$.

Lemma 2.1. Let $f, g \in A$ then convolution $(f * g) \in A$.

Proof: Let $f, g \in A$. From (2) and the definition of convolution $(f \star g)$ we have

$$
\begin{aligned}
\int_{0}^{\infty} \mid t^{\alpha-1}( & * g)(t) \mid d t \\
& =\int_{0}^{\infty}\left|t^{\alpha-1} \int_{0}^{t} e^{\frac{-i s}{\alpha}\left(x^{\alpha}-t^{\alpha}\right)} e^{\frac{-i s}{\alpha}(t-x)^{\alpha}}(t-x)^{\alpha-1} x^{\alpha-1} t^{1-\alpha} f(x) g(t-x) d x\right| d t \\
& =\int_{0}^{\infty}\left|e^{\frac{-i s}{\alpha} x^{\alpha}} x^{\alpha-1} f(x) \int_{x}^{\infty} e^{\frac{i s}{\alpha} t^{\alpha}} e^{\frac{-i s}{\alpha}(t-x)^{\alpha}}(t-x)^{\alpha-1} g(t-x) d t\right| d x \\
& =\int_{0}^{\infty}\left|e^{\frac{-i s}{\alpha} x^{\alpha}} x^{\alpha-1} f(x) \int_{0}^{\infty} e^{\frac{i s}{\alpha}(y+x)^{\alpha}} e^{\frac{-i s}{\alpha}(y)^{\alpha}} y^{\alpha-1} g(y) d y\right| d x
\end{aligned}
$$


Pravinkumar V. Dole and S.K.Panchal

$$
=\int_{0}^{\infty}\left|x^{\alpha-1} f(x)\right| \int_{0}^{\infty}\left|y^{\alpha-1} g(y)\right| d y d x \leq M_{1} M_{2}<M \text {, }
$$

where $\int_{0}^{\infty}\left|x^{\alpha-1} f(x)\right| d x \leq M_{1}, \int_{0}^{\infty}\left|y^{\alpha-1} g(y)\right| d y \leq M_{2}$ for some $M, M_{1}, M_{2}>0$.

Theorem 2.4. (Convolution theorem)

Let $f, g \in A$ and $\mathcal{P}_{\alpha}[f(t)](s)=F_{\alpha}(s), \mathcal{P}_{\alpha}[g(t)](s)=G_{\alpha}(s)$ then new fractional integral transform of convolution $(f g)$ is given by

$$
\mathcal{P}_{\alpha}[(f * g)(t)](s)=F_{\alpha}(s) G_{\alpha}(s) .
$$

Proof: Let $f, g \in A$. From the definition of new fractional integral transform and convolution $(f * g)$ we have,

$$
\begin{aligned}
\mathcal{P}_{\alpha}[(f * g)(t)](s) & =\int_{0}^{\infty} e^{\frac{-i s t^{\alpha}}{\alpha}} t^{\alpha-1} \\
& \times\left(\int_{0}^{t} e^{\frac{-i s}{\alpha}\left(x^{\alpha}-t^{\alpha}\right)} e^{\frac{-i s}{\alpha}(t-x)^{\alpha}}(t-x)^{\alpha-1} x^{\alpha-1} t^{1-\alpha} f(x) g(t-x) d x\right) d t \\
& =\int_{0}^{\infty} e^{\frac{-i s x^{\alpha}}{\alpha}} x^{\alpha-1} f(x) \int_{x}^{\infty} e^{\frac{-i s}{\alpha}(t-x)^{\alpha}}(t-x)^{\alpha-1} g(t-x) d t d x \\
& =\int_{0}^{\infty} e^{\frac{-i s x^{\alpha}}{\alpha}} x^{\alpha-1} f(x) \int_{0}^{\infty} e^{\frac{-i s}{\alpha}(y)^{\alpha}}(y)^{\alpha-1} g(y) d y d x=F_{\alpha}(s) G_{\alpha}(s) .
\end{aligned}
$$

Lemma 2.2. The space $(A, *)$ is commutative semigroup.

Proof: Let $f, g, h \in A$ and $(f * g)=u$ and $(g * h)=v$, we have to show that operation ' $*$ ' is commutative and associative in $A$.

(i) Commutativity

$$
\begin{aligned}
(f * g)(t) & =\int_{0}^{t} e^{\frac{-i s}{\alpha}\left(x^{\alpha}-t^{\alpha}\right)} e^{\frac{-i s}{\alpha}(t-x)^{\alpha}}(t-x)^{\alpha-1} x^{\alpha-1} t^{1-\alpha} f(x) g(t-x) d x \\
& =\int_{0}^{t} e^{\frac{-i s}{\alpha}\left(y^{\alpha}-t^{\alpha}\right)} e^{\frac{-i s}{\alpha}(t-y)^{\alpha}}(t-y)^{\alpha-1} y^{\alpha-1} t^{1-\alpha} f(t-y) g(y) d y \\
& =(g * f)(t) .
\end{aligned}
$$

(ii) Associativity

$$
\begin{aligned}
((f * g) * h)(t) & =(u * h)(t) \\
& =e^{\frac{i s}{\alpha} t^{\alpha}} t^{1-\alpha} \int_{0}^{t} e^{\frac{-i s}{\alpha} x^{\alpha}} e^{\frac{-i s}{\alpha}(t-x)^{\alpha}}(t-x)^{\alpha-1} x^{\alpha-1} u(x) h(t-x) d x \\
& =e^{\frac{i s}{\alpha} t^{\alpha}} t^{1-\alpha}(\hat{u} * \hat{h})(t)=e^{\frac{i s}{\alpha} t^{\alpha}} t^{1-\alpha}((\hat{f} * \hat{g}) * \hat{h})(t) .
\end{aligned}
$$

Similarly we have

$$
\begin{aligned}
(f *(g * h))(t) & =e^{\frac{i s}{\alpha} t^{\alpha}} t^{1-\alpha}(\hat{f} * \hat{v})(t) \\
& =e^{\frac{i s}{\alpha} t^{\alpha}} t^{1-\alpha}(\hat{f} *(\hat{g} * \hat{h}))(t) \\
& =e^{\frac{i s}{\alpha} t^{\alpha}} t^{1-\alpha}((\hat{f} * \hat{g}) * \hat{h})(t) .
\end{aligned}
$$

This implies $\quad((f * g) * h)(t)=(f *(g * h))(t)$. 
On a New Fractional Integral Transform and its Applications

\section{Applications}

In this section, we obtaine the solutions of diffusion equation and heat-transfer problem involving conformable fractional derivatives.

Problem 3.1. For $u \in \mathcal{L}^{1}\left(\mathbb{R}^{+}\right)$and $t>0$. The solution of the conformable fractional diffusion equation

is given by

$$
\begin{aligned}
& T_{\alpha} u(x, t)=\lambda u_{x x}(x, t) \\
& u(x, 0)=f(x)
\end{aligned}
$$

$$
u(x, t)=\sqrt{\frac{\alpha}{4 \pi \lambda t^{\alpha}}} \int_{-\infty}^{\infty} f(\xi) e^{\frac{-\alpha(x-\xi)^{2}}{4 \lambda t^{\alpha}}} d \xi
$$

Solution: Let $\bar{u}(x, s)$ and $\hat{u}(k, t)$ denote the new fractional integral transform and Fourier transform of $u(x, t)$ respectively. Taking new fractional integral transform and Fourier transform of (13) and using (8), (14), we get

$$
\begin{aligned}
& i s \overline{\hat{u}}(k, s)-F(k)=-\lambda k^{2} \overline{\hat{u}} \\
& \widehat{\bar{u}}(k, s)=\frac{F(k)}{i s+\lambda k^{2}} .
\end{aligned}
$$

Taking inverse new fractional integral transform and using (9),

$$
\hat{u}(k, t)=F(k) e^{\frac{-\lambda k^{2} t^{\alpha}}{\alpha}} \text { for } t>0 .
$$

Now using inverse Fourier transform we get,

$$
u(x, t)=\frac{1}{\sqrt{2 \pi}} \int_{-\infty}^{\infty} F(k) e^{\frac{-\lambda k^{2} t^{\alpha}}{\alpha}} d k
$$

From the convolution property of Fourier transform, we have

$$
\begin{gathered}
u(x, t)=\frac{1}{\sqrt{2 \pi}} \int_{-\infty}^{\infty} f(\xi) g(x-\xi) d \xi, \\
\text { where } g(x)=\mathcal{F}^{-1}\left[e^{\frac{-\lambda k^{2} t^{\alpha}}{\alpha}}\right]=\sqrt{\frac{\alpha}{2 \lambda t^{\alpha}}} e^{\frac{-\alpha x^{2}}{4 \lambda t^{\alpha}}} \text { for } t>0 .
\end{gathered}
$$

Finally, we see that the special case involving impulsive initial condition $u(x, 0)=\delta(x)$ and the property of delta function given by $\int_{-\infty}^{\infty} \delta(\xi) y(\xi) d \xi=y(0)$, the solution (15) reduces to

$$
u(x, t)=\sqrt{\frac{\alpha}{4 \pi \lambda t^{\alpha}}} e^{\frac{-\alpha x^{2}}{4 \lambda t^{\alpha}},} \quad t>0,
$$

where $e^{\frac{-\alpha(x-\xi)^{2}}{4 \lambda t^{\alpha}}}$ is a good function.

Problem 3.2 The solution of the conformable heat-transfer equation

$$
\begin{aligned}
-h M \theta(x) & =\rho V c_{p} T_{\alpha} \theta(x), \\
\theta(0) & =\beta,
\end{aligned}
$$

where $\rho$-density, $V$-volume, $c_{p}$-specific heat of material, $h$-convection heat transfer coefficient, $M$-surface area of the body and $\theta \in \mathcal{L}^{1}[0, \infty), 0<x<\infty ; \alpha \in(0,1]$ is given by, 
Pravinkumar V. Dole and S.K.Panchal

$$
\theta(x)=\beta e^{\left(\frac{-h M}{\rho V c_{p}}\right) \frac{t^{\alpha}}{\alpha}} .
$$

Proof: Taking the new fractional integral transform of (16) and using (8), (17) we get,

$$
\begin{aligned}
-h M \Theta_{\alpha}(s) & =\rho V c_{p}\left[i s \Theta_{\alpha}(s)-\beta\right] \\
\Theta_{\alpha}(s) & =\frac{\beta}{\left(i s+\frac{h M}{\rho V c_{p}}\right)}
\end{aligned}
$$

Taking the inverse new fractional integral transform of above equation and using (9) we get the required solution (18).

\section{Conclusion}

The new fractional integral transform is useful to solve the fractional differential equations involving conformable fractional derivative.

\section{REFERENCES}

1. L.Debnath, Recent applications of fractional calculus to science and engineering, International Journal of Mathematics and Mathematical Sciences, 54 (2003) 3413-3442.

2. D.S.Bodkhe and S.K.Panchal, On Sumudu transform of fractional derivatives and its application to fractional differential equations, Asian Journal of Mathematics and Computer Research, 11(1) (2016) 69-77.

3. B.Goodwine, Engineering Differential Equations: Theory and Applications. Springer, New York, USA, (2010).

4. R.Khalil, M.Al Horani, A.Yousef and M.Sababhehb, A new definition of fractional derivative, Journal of Computational and Applied Mathematics, 264 (2014) 65-70.

5. A.A.Kilbas, H.M.Srivastava and J.J.Trujillo, Theory and Applications of Fractional Differential Equations, Elsevier, Amsterdam, (2006).

6. K.B.Oldham and J.Spanier, The Fractional Calculus, Academic Press, New York, NY, USA, (1974).

7. S.K.Panchal, A.D.Khandagale and P.V.Dole, Sumudu transform of Hilfer-Prabhakar fractional derivatives with applications, Proceeding of National Conference on Recent Trends in Mathematics, 1 (2016) 60-66.

8. S.K.Panchal, Pravinkumar V. Dole and Amol D. Khandagale, k-Hilfer-Prabhakar fractional derivatives and its applications. Indian Journal of Mathematics(IJM), accepted for publication (2017).

9. I.Podlubny, Fractional Differential Equations. Academy Press, San-Diego, 1999.

10. S.C.Sharma and R.K.Bairwa, A reliable treatment of iterative laplace transform method for fractional telegraph equations, Annals of Pure and Applied Mathematics, 9(1) (2015) 81-89.

11. I.N.Sneddon, The Use of Integral Transforms, McGraw-Hill International Editions, New York, (1972). 